\title{
Dielectric and Theoretical Studies on L-histidinium 2-nitrobenzoate NLO Single Crystal
}

\author{
Sagadevan Suresh* \\ Crystal Growth Centre \\ Anna University
}

\begin{abstract}
A nonlinear optical single crystal of L-histidinium 2-nitrobenzoate has been grown by slow evaporation method. The grown crystal was subjected to single crystal X-ray diffraction analysis to confirm that the crystal belongs to the monoclinic system. Dielectric studies reveal that both dielectric constant and dielectric loss decreases with increase in frequency at different temperatures. Some fundamental data such as valance electron plasma energy, Penn gap, Fermi energy and electronic polarizability of the grown crystal were calculated.
\end{abstract}

\section{Keywords}

Crystal growth, Slow evaporation method, Single crystal XRD, Dielectric studies

\section{INTRODUCTION}

Research in organic and inorganic functionalized nonlinear optical materials plays a crucial role because of their molecular interactions, bond strength, high molecular polarizability, easy incorporation of ions in the lattice, etc. [1] and [2]. Nonlinear optical (NLO) material capable of generating the second harmonic frequency plays an important role in the domain of optoelectronics and photonics. Within the last decade, much progress has been made in the development of these NLO organic materials having large nonlinear optical coefficient. In the recent years, there have been extensive efforts to develop inorganic, organic and semi organic NLO single crystals [3-6]. The key factor for material selection depends not only on laser conditions but also on the physical properties of the crystal, such as transparency, damage threshold, conversion efficiency, phase matching and temperature stability $[7,8]$. Also, microelectronic industries require crystals possess low dielectric constant at higher frequency [9]. Keeping this view, attempts have been made to grow and study the optical, electrical and mechanical properties of crystals. Naturally, many kinds of the nonlinear optical crystals have soft nature and so are difficult to polish or cut the crystals to meet the requirements in the fabrication of optical devices. To avoid this problem in these materials, inorganic or organic acid is added to amino acids for improving optical nonlinearity, mechanical and thermal behavior of the crystals. Solution growth is a versatile technique which can be used to synthesis and grow new nonlinear optical materials and so it stimulates considerable interest among the researchers in the field of crystal growth. Hence, it may be useful to prepare semi-organic crystals which combine the positive aspects of organic and inorganic materials resulting in useful nonlinear optical properties [10]. One of the essential requirements for a material to exhibit second order optical susceptibilities described by third rank polar tensor is that the crystal should have non- centrosymmetric space group. The research on the synthesis of organic and inorganic complexes increased enormously in the last few years [11-13]. Specifically, amino acids and strong inorganic acids are good raw materials to produce semi-organic crystals because amino acid crystals are having good optical properties [14]. Amino acids are interesting materials for NLO applications as they contain a proton donor carboxyl group $(\mathrm{COOH})$ and the proton acceptor amino $\left(\mathrm{NH}_{2}\right)$ group in them [15]. In the present work, we have grown the L-histidinium 2-nitrobenzoate single crystal, studied the cell parameters using the single crystal XRD. Furthermore, the variation of dielectric constant and dielectric loss with frequency at different temperatures were also investigated. Some fundamental data such as valance electron plasma energy, Penn gap, Fermi energy and electronic polarizability of the grown crystal were calculated.

\section{EXPERIMENTAL DETAILS}

L-histidinium 2-nitrobenzoate single crystals were synthesized by dissolving L-histidine and 2-nitrobenzoic acid in the molar ratio 1:1 in distilled water. The solution was stirred continuously using magnetic stirrer. The prepared solution was filtered and kept undisturbed at room temperature. Tiny seed crystals with good transparency were obtained due to the spontaneous nucleation. Among them, defect free seed crystal was suspended in the mother solution, which was allowed to evaporate at room temperature. Large size single crystals were obtained due to collection of monomers at the seed crystal sites from the mother solution.

\section{SINGLE CRYSTAL XRD}

Single crystal X-ray diffraction study was carried out on the as grown L-histidinium 2-nitrobenzoate crystal. The calculated unit cell parameters, presented in Table 1 are in good agreement with the corresponding reported value of Subramanian Natarajan et al [16].

Table 1. Comparison of single crystal X-ray data of L-histidinium 2-nitrobenzoate crystal

\begin{tabular}{|c|c|c|}
\hline Parameters & Present work & Subramanian Natarajan et al \\
\hline $\mathrm{a}(\mathbf{A})$ & 5.13 & 5.147 \\
\hline $\mathrm{b}(\stackrel{\AA}{\mathbf{A}})$ & 7.23 & 7.228 \\
\hline $\mathrm{c}(\stackrel{\AA}{\mathbf{A}})$ & 18.89 & 18.887 \\
\hline System & Monoclinic & Monoclinic \\
\hline$\beta\left(^{\circ}\right)$ & 92.62 & 92.72 \\
\hline Space group & $\mathrm{P} 2_{1}$ & $\mathrm{P} 2_{1}$ \\
\hline
\end{tabular}




\section{DIELECTRIC PROPERTIES}

The dielectric characteristics of the materials are important to study the lattice dynamics in the crystal. Hence, the cut and polished grown single crystal of L-histidinium 2nitrobenzoate was used for dielectric studies. The surface of the sample was electroded with silver paste for electrical contact. The experiment was carried out for the frequencies from $50 \mathrm{~Hz}$ to $5 \mathrm{MHz}$ with the temperatures $30^{\circ} \mathrm{C}, 60^{\circ} \mathrm{C}, 90$ ${ }^{\circ} \mathrm{C}, 120^{\circ} \mathrm{C}$ and $150{ }^{\circ} \mathrm{C}$ using HIOKI 3532 LCR HITESTER. The dielectric constant $\varepsilon_{\mathrm{r}}$ was calculated using the relation,

$$
\varepsilon_{r}=C d / \varepsilon_{0} A
$$

where $C$ is the capacitance, $d$ is the thickness of the crystal, $\varepsilon_{\mathrm{o}}$ is the vacuum dielectric constant and $\mathrm{A}$ is the area of the crystal. The dielectric loss was calculated using the relation

$$
\varepsilon^{\prime \prime}=\varepsilon_{r} D
$$

where $D$ is the dissipation factor. Figs. 1 and 2 show the dielectric constant $\left(\varepsilon_{\mathrm{r}}\right)$ and the dielectric loss for different points, respectively. From the figs, it is found that the values of dielectric constant and dielectric loss decreases with the increase in frequency and dielectric constant of crystal at higher frequencies is almost constant. The very high value of $\varepsilon_{\mathrm{r}}$ at low frequencies may be due to the presence of all the four polarizations, namely space charge, orientional, ionic and electronic polarizations and its low value at high frequencies may be due to the loss of significance of these polarizations gradually [17]. The value of dielectric loss at high frequencies reveals the high optical quality of the crystal with lesser defects, which is the desirable property for NLO applications [18].

\section{CHARACTERISTIC DATA OF THE L-HISTIDINIUM 2-NITROBENZOATE SINGLE CRYSTAL}

Theoretical calculations shows that the high frequency dielectric constant is explicitly dependent on the valence electron Plasmon energy, an average energy gap referred to as the Penn gap and the Fermi energy. The Penn gap is determined by fitting the dielectric constant with the Plasmon energy [19]. The total number of valence electrons of L-histidinium 2-nitrobenzoate $\mathrm{Z}=122$, density $\rho=1.525$ $\mathrm{gcm}^{-3}$ and molecular mass $\mathrm{M}=322.28$, the valence electron plasma energy, $\hbar \omega_{p}$, is calculated using the relation,

$$
\begin{gathered}
\hbar \omega_{P}=28.8\left(\frac{Z \rho}{M}\right)^{1 / 2} \\
E_{P}=\frac{\hbar \omega_{P}}{\left(\varepsilon_{\infty}-1\right)^{1 / 2}}
\end{gathered}
$$

Plasma energy are the Penn gap and the Fermi energy [19] given by

$$
E_{F}=0.2948\left(\hbar \omega_{P}\right)^{4 / 3}
$$

Then we obtained electronic polarizability $\alpha$ using a relation $[20,21]$,

$\alpha=\left[\frac{\left(\hbar \omega_{P}\right)^{2} S_{0}}{\left(\hbar \omega_{P}\right)^{2} S_{0}+3 E_{P}^{2}}\right] \times \frac{M}{\rho} \times 0.396 \times 10^{-24} \mathrm{~cm}^{3}$

where $S_{0}$ is a constant given by

$$
S_{0}=1-\left[\frac{E_{P}}{4 E_{F}}\right]+\frac{1}{3}\left[\frac{E_{P}}{4 E_{F}}\right]^{2}
$$

The value of $\alpha$ obtained from equation (6) closely matches with that obtained using Clausius-Mossotti relation,

$$
\alpha=\frac{3}{4} \frac{M}{\pi N_{a} \rho}\left[\frac{\varepsilon_{\infty}-1}{\varepsilon_{\infty}+2}\right]
$$

All the above parameters as estimated are shown in Table 2.

Table.2 Important parameters of L-histidinium 2-nitrobenzoate Single crystal

\begin{tabular}{|c|c|}
\hline Parameters & Value \\
\hline Plasma energy $\left(\mathrm{h} \omega_{\mathrm{p}}\right)$ & $21.88 \mathrm{eV}$ \\
\hline Penn gap $\left(\mathrm{E}_{\mathrm{p}}\right)$ & $4.06 \mathrm{eV}$ \\
\hline Fermi Energy $\left(\mathrm{E}_{\mathrm{F}}\right)$ & $17.86 \mathrm{eV}$ \\
\hline $\begin{array}{c}\text { Electronic polarizability } \\
\text { (Penn analysis) }\end{array}$ & $7.541 \times 10^{-23} \mathrm{~cm}^{-3}$ \\
\hline $\begin{array}{c}\text { Electronic polarizability } \\
\text { (using CM relation) }\end{array}$ & $7.595 \times 10^{-23} \mathrm{~cm}^{-3}$ \\
\hline
\end{tabular}




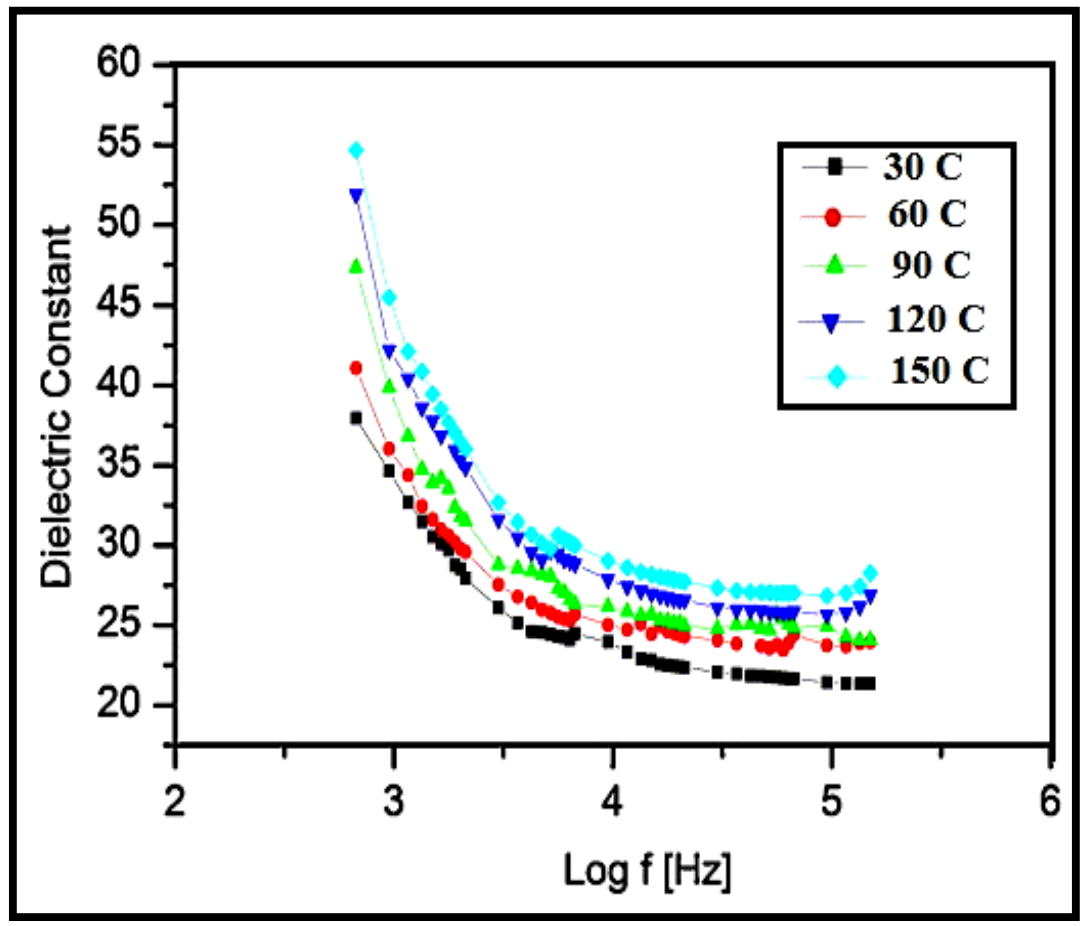

Fig.1. Variation of dielectric constant of L-histidinium 2-nitrobenzoate with log frequency

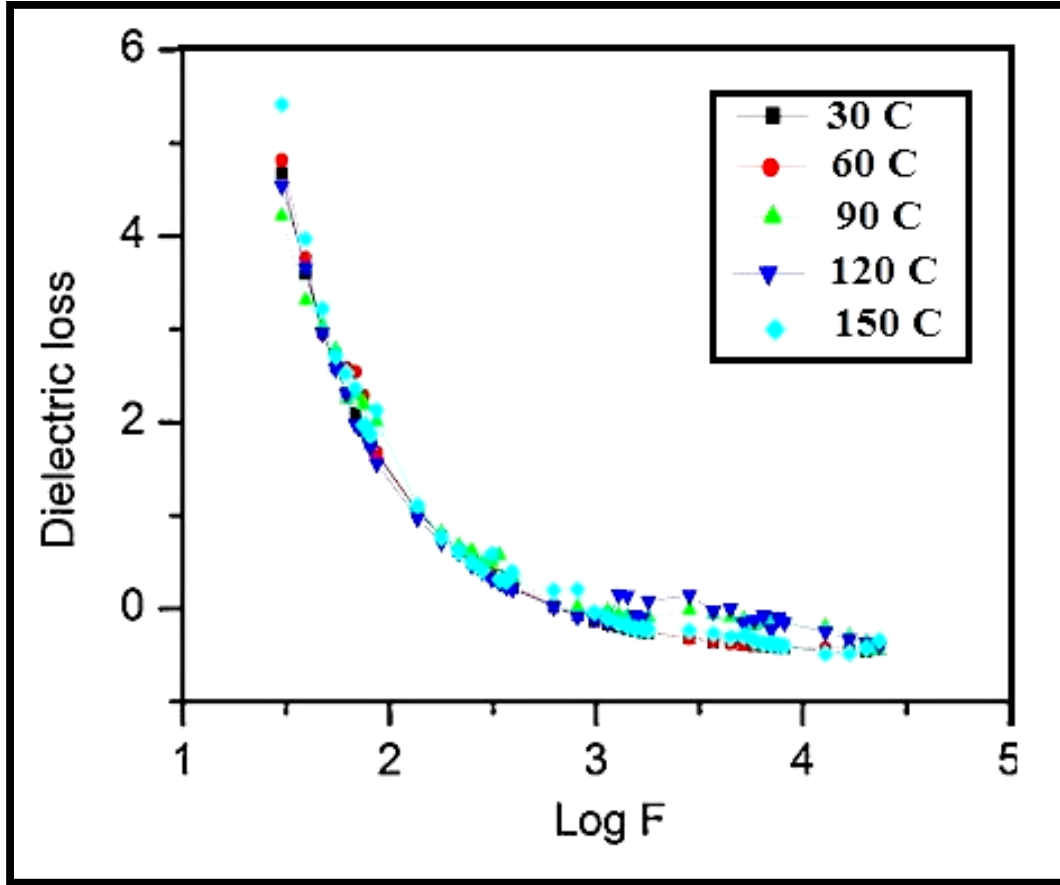

Fig .2. Variation of dielectric loss of L-histidinium 2-nitrobenzoate with log frequency 


\section{CONCLUSION}

Single crystals of nonlinear optical L-histidinium 2-nitrobenzoate were grown by slow evaporation method. The grown crystals were characterized by single crystal XRD analysis and dielectric properties. Single crystal XRD study reveals that the L-histidinium 2-nitrobenzoate crystal belongs to monoclinic structure and space group $\mathrm{P} 2{ }_{1}$. The space group natures clearly confirm the non-centrosymmetric nature of the grown crystals from the XRD studies. Dielectric properties were carried out by using parallel capacitor method and the results indicate that dielectric constant and dielectric loss decreases with increase in frequency and temperature. Dielectric constant and dielectric loss of the crystal are low at high frequency region. Theoretical calculations shows that the high frequency dielectric constant is used to calculate the solid state parameters such as plasma energy, Penn gap, Fermi energy and polarizability were calculated.

\section{REFERENCES}

[1] Cong Zhang, Zhen Li, Hengjiang Cong, Jiyang Wang, Huaijin Zhang, R.I. Boughton, 2010, J. Alloys Compd. $507,335$.

[2] Mark J, Patricia Cunningham Rosker, Mark D. Ewbank, Henry O. Marcy, Frederick R. Vachss, Leslie F. Warren, Robert Gappinger, Robert Borwick,1996, Pure Appl. Opt. 5,667 .

[3] Xiue Ren, Dongli Xu, Dongfeng Xue, 2008, J. Cryst. Growth. 310, 2005-2009.

[4] Dongfeng Xue, Henryk Ratajczak, 2003, Chem Phys. Lett. 371, 601-607.

[5] Dongli Xu, Dongfeng Xue, 2008, J. Cryst. Growth. 310, 1385-1390.

[6] Dongfeng Xue, Siyuan zhang, 199, Chem. Phys. Lett. 310, 449-452.
[7] Zernike F., Midwinter, 1973, J.E. in: Applied Nonlinear Optics, Wiley, New York

[8] Prasad PN, Willams DJ, 1991, in: Introduction to Nonlinear Effects in molecules and Polymers, Wiley, New York

[9] Hatton BD, Landskron K, Hunks WJ, Bennett MR, Shukaris D, Perovic DD and Ozin GA,2006, Mater. Today. 9,22-31

[10] Muralitharan R, Mohankumar R, Jayavel R and Ramasamy P, 2003, J. Cryst. Growth. 259, 321-325.

[11] Adhikari S and Kar T, 2012, J. Cryst. Growth, 356, 4-9.

[12] Koshima H, Hamada M, Yagi I and Uosaki K., 2001, Cryst. Growth Des. 1, 467-471.

[13] Prakash, M. Geetha, D. and LydiaCaroline, M, 2011 , Physica B. 406, 2621-2625

[14] C. Razzeti, M.Ardoino, L.Zanotti, M.Zha, C.Paorici,2002, Cryst.Res.Technol. 456

[15] Pal T, Kar T, Bocelli G and Rigi L, 2003, Cryst. Growth Des, 3,13-16.

[16] Subramanian Natarajan, Kalimuthu Moovendaran, Jeyaperumal Kalyana Sundar and Krishnan Ravikumar, 2012, Journal of Amino Acids. 1-6

[17] Prasad NV, Prasad G, Bhimasankaran T, Suryanarayana SV, Kumar GS, 1996, Indian J. Pure Appl. Phys. 34, 639.

[18] Balarew C, Duhlew R, 1984, J. Solid State Chem. 55, 1

[19] Ravindra NM, Bharadwaj RP, Sunil Kumar K and Srivastava VK, 1981, Infrared Phys. 21,369

[20] Ravindra NM and Srivastava VK, 1980, Infrared Phys. 20,67

[21] Penn DR, 1962, Phys. Rev. 128, 2093 